\title{
Die Testierfähigkeit von Menschen mit Demenz
}

\section{Brigitte Rüegger-Frey ${ }^{a, f}$, Georg Bosshard ${ }^{b, f}$, Daniel Grob ${ }^{c, f}$, Peter Breitschmide,f, Sacha Beck ${ }^{d, f}$}

a lic. phil. Fachpsychologin für Neuropsychologie FSP, Gutachterin SIM, Zürich; b PD Dr. med., Facharzt für Innere Medizin, spez. Geriatrie, Winterthur; ${ }^{c}$ Dr. med. MHA, Facharzt für Innere Medizin, spez. Geriatrie, Zürich; ${ }^{d}$ Dr. med. MHA, Facharzt für Innere Medizin, spez. Geriatrie, cand. Gutachter SIM, Zürich; ${ }^{e}$ Prof. Dr. iur., Emeritus UZH Lehrstuhl für Privatrecht mit Schwerpunkt Zivilgesetzbuch, Zürich; ${ }^{\dagger}$ Fachstelle Urteilsfähigkeit im Alter, Zürich

Um gemäss Zivilgesetzbuch ein Testament errichten zu können, muss eine Person «mindestens 18 Jahre alt und urteilsfähig sein» (= Testierfähigkeit). Der ärztlichen Einschätzung der Urteilsfähigkeit von Menschen mit einer Demenz kommt deshalb eine zentrale Bedeutung zu. Um das Risiko von Ungültigkeitsklagen post mortem wegen (behaupteter) Urteilsunfähigkeit zu minimieren, schlagen die Autoren in komplexen Situationen ein mehrstufiges und interdisziplinäres Vorgehen vor.

Ein Testament kann jederzeit selber verfasst werden und ist gültig, wenn es von Hand geschrieben, datiert und unterschrieben wird. Anfechtungsklagen drohen, wenn nach dem Tod des Erblassers der Verdacht auf eine Demenz aufkommt, und es zu Streitigkeiten um die Urteilsfähigkeit kommt. Gemäss Art. 16 ZGB ist «jede Person urteilsfähig, der nicht wegen ihres Kindesalters, infolge geistiger Behinderung, psychischer Störung, Rausch oder ähnlicher Zustände die Fähigkeit mangelt, vernunftgemäss zu handeln». Der Gesetzgeber geht also davon aus, dass Urteilsfähigkeit grundsätzlich «vermutet» werden kann und Urteilsunfähigkeit bewiesen werden muss. Auch wenn ein sorgfältiges und vertieftes Vorgehen die Wahrscheinlichkeit einer

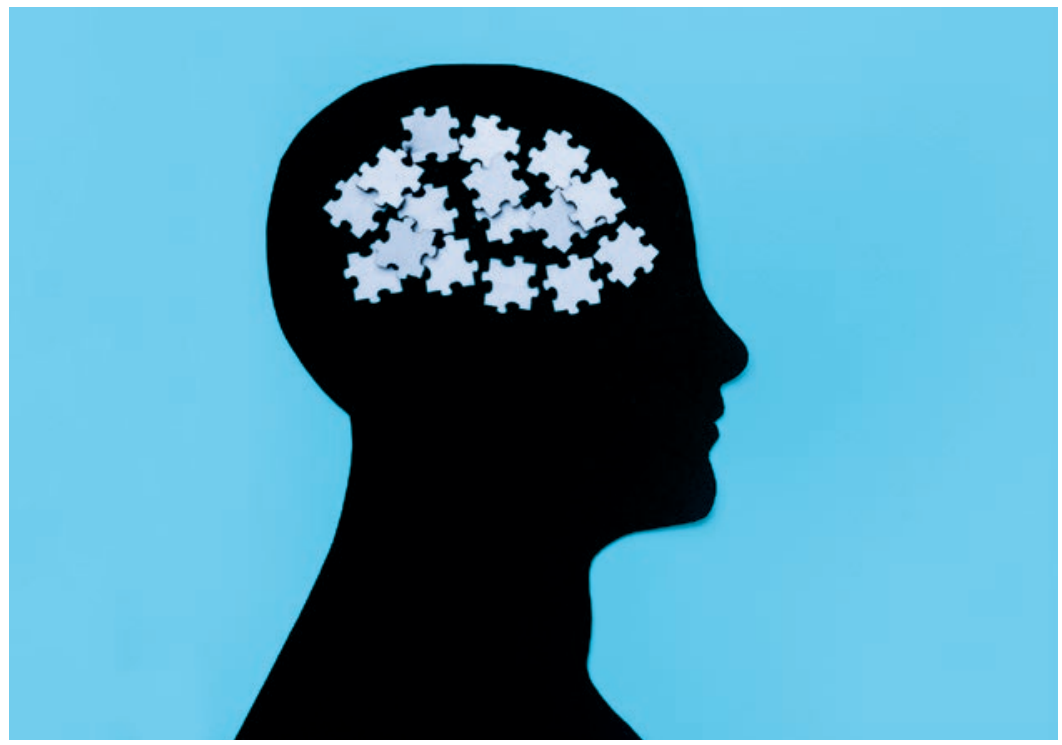

Wenn die Dinge beginnen durcheinanderzugeraten: Eine Demenzerkrankung kann die Urteilsfähigkeit tangieren. korrekten Beurteilung der Testierfähigkeit erhöht, bestehen oft Restunsicherheiten. Testierfähigkeit ist schlussendlich immer entweder gegeben oder nicht, es gibt keine Graustufen. Im Streitfall entscheidet der Zivilrichter unter Würdigung der vorliegenden Beweise.

\section{Gerichtliche «Umkehrung» der vermute- ten Urteilsfähigkeit}

Die grundsätzlich vermutete Urteilsfähigkeit kann in speziellen Situationen vom Gericht «umgekehrt» werden. Nämlich dann, wenn «die allgemeine Lebenserfahrung» den Schluss zulässt, dass die Urteilsfähigkeit nicht mehr gegeben ist. Bei einer Patientin mit einer fortgeschrittenen Demenzerkrankung gilt in einem Streitfall also eine umgekehrte Beweislast. Die Urteilsfähigkeit muss bewiesen werden [1].

Für die Praxis ist es deshalb wichtig, Menschen mit kognitiven Einschränkungen früh abzuklären, Vorsorgeregelungen frühzeitig anzugehen und die Urteilsfähigkeit "zu Lebzeiten" sauber und nachvollziehbar darzulegen. Nur so können die Wünsche und der Wille von Menschen mit dementiellen Erkrankungen auch gegenüber klagenden Dritten durchgesetzt werden.

\section{Eine Demenzdiagnose bedeutet nicht Urteilsunfähigkeit}

Bei der Definition von Urteilsfähigkeit gemäss Art. 16 ZGB liegt laut Wolf eine juristische Betrachtungsweise im Vordergrund. Relevant ist also nicht die Demenzdiagnose alleine, sondern primär «deren Auswirkung auf die Fähigkeit, vernunftgemäss zu handeln» [2]. Das 
Vorliegen einer Demenzdiagnose reicht aus diesem Grund nicht aus, um eine abschliessende Aussage über die Testierfähigkeit zu machen. Vielmehr geht es darum, eine Gegenüberstellung zu machen, welchen Anforderungen der Erblasser für die Entscheidungsfindung genügen muss und auf welche kognitiven Fähigkeiten er dabei zählen kann. Die Überprüfung der Testierfähigkeit ist deshalb eine anspruchsvolle Tätigkeit und bedarf juristischen Wissens [1].

\section{Relativität und Dichotomie}

Die Urteilsfähigkeit muss immer für ein bestimmtes Geschäft und zu einem bestimmten Zeitpunkt abgeklärt werden (sachliche und zeitliche Relativität). Zudem gibt es im Zivilrecht keine Abstufungen der Urteilsfähigkeit, sie ist also entweder gegeben oder nicht (Dichotomie). Das Bundesgericht verneint bei der Erstellung von Testamenten nur sehr zurückhaltend die Urteilsfähigkeit. Dies wird neben dem Grundsatz, dass Urteilsfähigkeit vermutet wird, damit begründet, dass Erblasser nicht im besonderen Masse geschützt werden müssten, weil sie die Auswirkungen ihres Testamentes nicht mehr erleben [1]. Das Damoklesschwert der Anfechtung belastet aber die Erben und ist ein kostspieliger Konfliktherd.

\section{Notarielle Beurkundung schützt nicht vor Anfechtung}

Eine notarielle Beurkundung schützt nicht vor einer Anfechtung durch Dritte bzw. entledigt die Ärztin nicht von der Aufgabe, selber eine ausgewogene Einschätzung der Urteilsfähigkeit abzugeben. Notare haben eine Urkundspflicht und dürfen von Gesetzes wegen von der Urteilsfähigkeit ihrer Mandanten und Mandantinnen ausgehen. Bestehen "Zweifel» an der Urteilsfähigkeit, dürfen sie die Beurkundung ablehnen und sollen auch weitere Abklärungen vornehmen [2]. Wie diese «Zweifel» aussehen müssen, bleibt allerdings unklar. Wenn man berücksichtigt, dass gut die Hälfte der Demenzerkrankungen nicht oder sehr spät diagnostiziert werden, kann vermutet werden, dass die Dunkelziffer der unentdeckten Urteilsunfähigkeit gross ist.

\section{Abklärung durch den Hausarzt - anspruchsvoll und zeitaufwändig}

In fraglichen Fällen kann der Notar seine Mandantin bitten, beim Hausarzt die Urteilsfähigkeit bescheinigen zu lassen. Mittlerweile existieren medizinisch- ethische Leitlinien [3, 4] als Orientierungshilfe für die medizinische Praxis, auch wenn diese primär für die Einwilligungsfähigkeit in medizinische Behandlungen ausgelegt sind. Drei Faktoren machen die Beurteilung der Testierfähigkeit in der Hausarztpraxis anspruchsvoll: 1. die notwendige Expertise im Feld der dementiellen Erkrankungen und deren (potentiellen) Auswirkungen auf die Urteilsfähigkeit, 2. die Notwendigkeit von rechtlichem und inhaltlichem Wissen in Bezug auf das zu beurkundende Testament und 3. das Aufbringen (und auch die Finanzierung) der dazu notwendigen Zeitressourcen. Zudem besteht die Gefahr, dass die Gerichte bei einer Anfechtungsklage von einer zu positiven Einschätzung des langjährigen Hausarztes ausgehen und die Bestätigung der Urteilsfähigkeit als «Gefälligkeit» auslegen könnten.

\section{Objektivierbarkeit gewährleisten}

Die Beurteilung der Urteilsfähigkeit beim Hausarzt gewinnt an Objektivität, wenn Screeninginstrumente (z.B. Minimental Status, Uhrentest, MoCa-Test) eingesetzt und Befunde detailliert erhoben und protokolliert werden. Screeninginstrumente besitzen aber zur Diagnosestellung der Demenz und zur Beurteilung der Urteilsfähigkeit nur eine begrenzte Aussagekraft und sind deshalb alleine ungenügend für eine abschliessende Beurteilung [1,5-8]. Sie sind also in der Beurteilung der Urteilsfähigkeit lediglich ergänzend einzusetzen.

\section{Abgestufte und interdisziplinäre Überprüfung}

Bei komplexen Erbschaftsverhältnissen empfehlen die Autoren eine mehrstufige und interdisziplinäre Prüfung der Testierfähigkeit (Abb. 1).

\section{Stufe 1: kognitiver "Work Up»}

Eine Gedächtnisstörung muss nicht immer das Erstsymptom einer Demenzerkrankung sein. Ein Delir gemäss ICD-10 [9], das typischerweise fluktuierend verläuft, «luzide» Intervalle haben, und auch bei kognitiv gesunden Menschen im Rahmen schwerer internistischer Erkrankungen vorkommen kann, ist von einer dementiellen Erkrankung möglichst exakt abzugrenzen. Gleiches gilt für eine Depression. Begleiterkrankungen wie zum Beispiel eine Suchtproblematik oder die Einnahme von Medikamenten sind zu berücksichtigen. Bestehen Verdachtsmomente für eine eingeschränkte Urteilsfähigkeit, ist eine kognitive Standortbestimmung angezeigt, die sich an die gültigen Abklärungsempfehlungen anlehnen soll [6]. 
Stufe 2: Abgleich kognitiver Einschränkungen mit Ressourcen und Kompensationsmöglichkeiten

Für die Testierfähigkeit sind neurokognitive Grundfunktionen wie das Gedächtnis, die Aufmerksamkeit, die Sprache und im Besonderen auch die exekutiven Funktionen (logisches Denken, Planen, Abstrahieren) wesentlich. Zudem sollten Persönlichkeitsveränderungen, Denkstörungen und die emotionale Befindlichkeit abgeklärt werden. Die Beurteilung findet aus Sicht der Autoren idealerweise interdisziplinär in Zusammenarbeit mit Neuropsychologinnen, Ärztinnen und auch Experten der Rechtsanwendung statt. Die Inter- disziplinarität hat den grossen Vorteil, dass gemäss den kognitiven Einschränkungen und Ressourcen des Erblassers eine inhaltliche und formale Prüfung des Testamentes vorgenommen werden kann. Wenn möglich und nötig können auch Anpassungen in der Verständlichkeit des Testaments vorgeschlagen werden, sofern diese zur Gewährleistung der Testierfähigkeit beitragen können und weiterhin den Wünschen des Erblassers entsprechen.

\section{Stufe 3: Das Gespräch über das Testament}

Die Abklärung einer allenfalls vorhandenen Demenzerkrankung und die Überprüfung mentaler Grund-

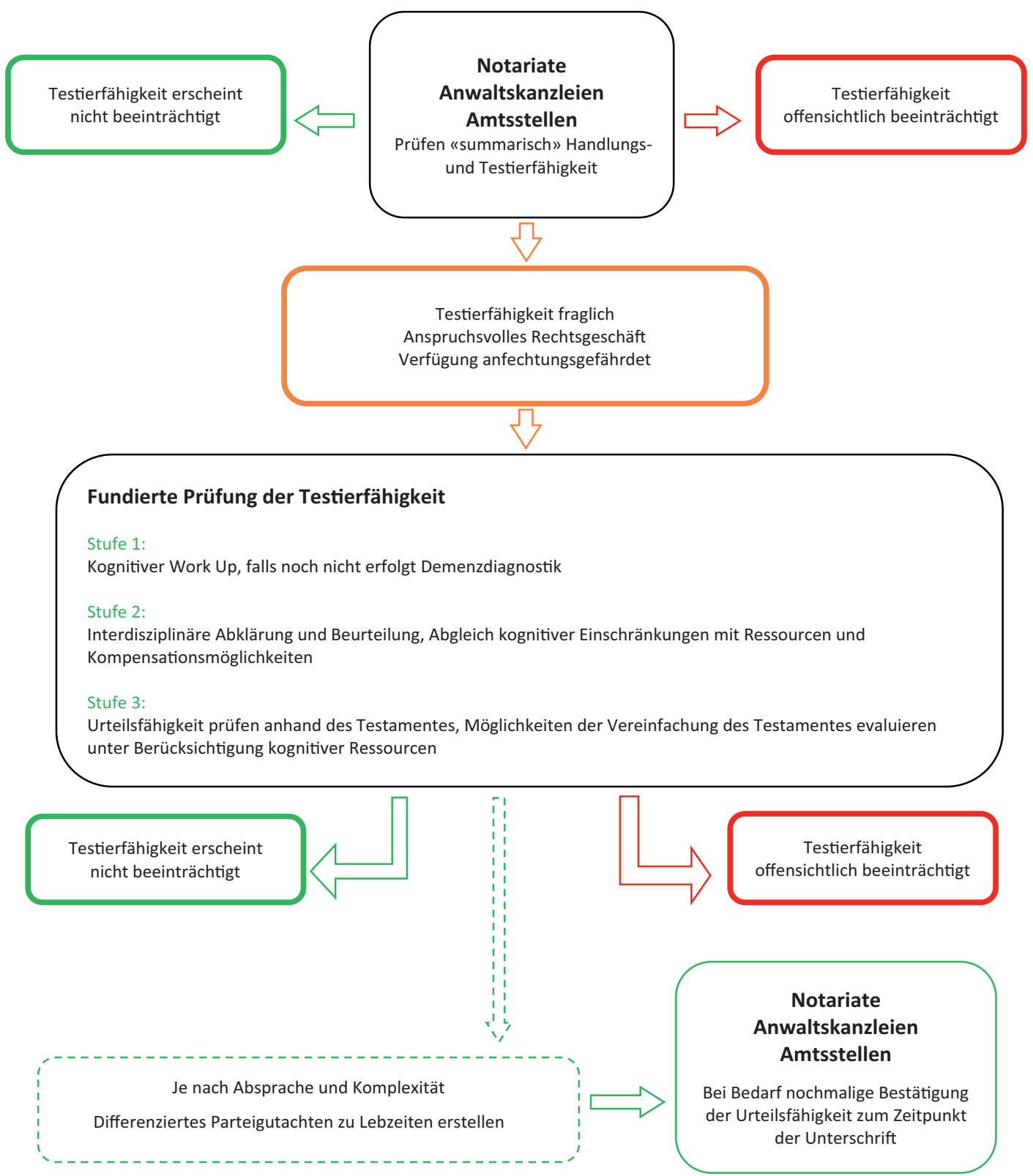

Abbildung 1: Vereinfachte Darstellung möglicher Entscheidungswege im Rahmen der Beurteilung der Testierfähigkeit in komplexen Situationen. In einfachen Situationen genügt normalerweise die hausärztliche Prüfung der Urteilsfähigkeit. 
funktionen mit Kompensationsmöglichkeiten bilden eine notwendige Voraussetzung, genügen aber nicht, um die Testierfähigkeit abschliessend zu beurteilen. Dazu ist immer auch die detaillierte Auseinandersetzung mit dem eigentlichen Inhalt des Testamentes nötig. Für die Abklärung sollte ein vertrauter Rahmen gewählt werden und die Gesprächstechnik sollte sich der Aufnahmefähigkeit der Betroffenen anpassen. Empeinfache Worte zu fassen.

\section{Urteilsfähigkeit: Entscheidungsprozess ist massgebend}

Das Kernstück der Überprüfung stellt in Analogie zu medizinischen Fragestellungen ein semistrukturiertes Interview unter Berücksichtigung der Erkenntnis-, Wertungs-, Willensbildungs- und der Willensumsetzungsfähigkeit dar [2,3,10].

Erkenntnisfähigkeit: Fähigkeit, die Testamentssituation zu erfassen. Minimales Wissen, was ein Testament bewirken kann. Kenntnis über die ungefähre Höhe und Zusammensetzung des Vermögens. Grundwissen über die gesetzliche Erbfolge und mögliche Abweichung durch das Testament. Wissen, bei wem unabhängiger Rat eingeholt werden kann.

Wertungsfähigkeit: Persönliche Bedeutung des Testaments abwägen. «Blick aufs Ganze» haben. Wer soll bedacht und wer ausgeschlossen werden und warum. Fähigkeit, seine Anordnungen in den Kontext seiner Biographie, Lebensumstände und seiner Beziehungen zu setzen.

Willensbildungsfähigkeit: Eigene Entscheidungen treffen, kommunizieren und vertreten. Aufgrund der persönlichen Erfahrung, dem «roten Lebensfaden», der Wertvorstellungen und im Kontext der Lebensumstände handeln. Konstanz und Konsistenz im Entscheid haben.

\section{Das Wichtigste in Kürze}

- Aufgrund der Demographie werden dementielle Erkrankungen zunehmen, viele Betroffene sind nicht korrekt abgeklärt, weshalb es bei der Beurteilung der Testierfähigkeit zu Fehleinschätzungen kommen kann. Wichtig festzuhalten ist, dass eine Demenzdiagnose nicht mit dem Wegfall der Urteilsfähigkeit gleichgesetzt werden kann.

- Urteils- undTestierfähigkeit sind rechtlich entweder gegeben oder nicht, es gibt keine Graustufen. Der richtige Umgang mit Unsicherheiten in der Beurteilung ist deshalb zentral.

Sacha Beck, Dr. med. MHA Innere Medizin, spez. Geriatrie Fachstelle Urteilsfähigkeit im Alter, Zürich sacha.beck[at]hin.ch

- Bei komplizierten Testamenten oder bei unklaren kognitiven Ressourcen des Erblassers kann eine mehrstufige, interdisziplinäre Abklärung sinnvoll sein, um die Betroffenen vor dem Risiko von Ungültigkeitsklagen wegen (behaupteter) Urteilsunfähigkeit schützen. Im Streitfall entscheidet der Zivilrichter. fehlenswert ist es auch, das Testament in möglichst

Willensumsetzungsfähigkeit: Stabiler Entschluss, den Willen durchzusetzen. Meinungen und Einflüsse von Dritten können kritisch abgewogen und wo nötig abgewehrt werden. Keine fremde Beeinflussung.

Weil Menschen mit kognitiven Einschränkungen oft in Abhängigkeitsverhältnissen leben (müssen), ist die Abgrenzung zwischen «Unterstützung» und «Beeinflussung» oft schwierig.

\section{Bildnachweis}

Symbolbild, @ Ilia Burdun | Dreamstime.com

Literatur

1 Aebi-Müller R. Testierfähigkeit im Schweizerischen Erbrecht - unter besonderer Berücksichtigung der bundesgerichtlichen Praxis. successio. 2012;1:4-32.

2 Wolf St, Nuspliger I. Die Urteilsfähigkeit aus rechtlicher Sicht insbesondere ihre Prüfung durch den Notar. Therapeutische Umschau. 2015;72(4):247-53.

3 Medizinisch-ethische Richtlinien. Urteilsfähigkeit in der medizinischen Praxis. Schweizerische Akademie der Medizinischen Wissenschaften (SAMW). 2019.

4 Einwilligung von Menschen mit Demenz in medizinische Massnahmen. Interdisziplinäre S2k-Leitlinie für die medizinsche Praxis. AWMF-Leitlinien Registernummer 108-001. 2020.

5 Breitschmid P. Über die Urteilsunfähigkeit des Urteilsfähigen und die Urteilsfähigkeit des Urteilsunfähigen. Thesen zur Urteilsfähigkeit aus rechtlicher Sicht. In Petermann FTH (eds). Urteilsfähigkeit. Schriftenreihe des Instituts für Rechtswissenschaft und Rechtspraxis. 2014;90:91-119.

6 Bürge M, Bieri G, Brühlmeier M, Colombo F, Demonet JF, Felbecker A, et al.Die Empfehlungen der Swiss Memory Clinics für die Diagnostik der Demenzerkrankungen. Praxis. 2018;107(8):435-51.

7 Jahn T. Neuropsychologische Gutachten in zivilrechtlichen Verfahren. Möglichkeiten und Grenzen psychometrischer Untersuchungsmethoden. Foren Psychiatr Psychol Kriminol. 2017;11:213-27.

8 Cording C, Nedopil N. Psychiatrische Begutachtung im Zivilrecht. Ein Handbuch für die Praxis. Lengerich: Pabst Science Publishers; 2014.

9 Deutsches Institut für Medizinische Dokumentation und Information. Internationale statistische Klassifikation der Krankheiten und verwandter Gesundheitsprobleme. 10. Revision. German Modification (ICD-10-GM). Version 2019.

10 Schweizerische Akademie der medizinischen Wissenschaften, Universität Zürich. U-Doc: Evaluation der Urteilsfähigkeit. 2018.

\section{L'essentiel en bref}

- Les cas de démence augmentent avec l'évolution démographique, mais ne sont pas tous bien diagnostiqués. Cela peut mener à des évaluations erronées de la capacité d'une personne à rédiger un testament. Un diagnostic de démence ne doit pas être assimilé à la perte de la capacité de discernement.

- D'un point de vue juridique, les capacités de discernement et testamentaire sont présentes ou absentes. Il est donc central de tenir compte des incertitudes dans l'évaluation.
- En cas de testaments compliqués ou si les capacités cognitives du testateur font I'objet d'hésitations, une évaluation interdisciplinaire par étape peut permettre de protéger les personnes concernées contre le risque d'actions en annulation pour cause d'incapacité (présumée) de discernement. Le juge civil tranche en cas de litige. 